\title{
Los frustrativos como aspecto: Un análisis a partir del chorote (mataguayo) y el mẽbengokre (jê)
}

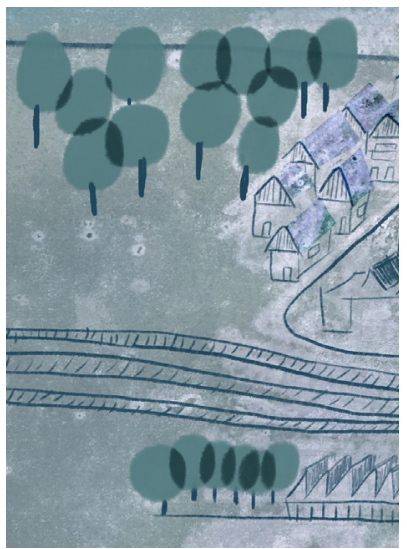

Javier Carol

Consejo Nacional de Investigaciones Científicas y Técnicas, Argentina / Universidad Autónoma de Entre Ríos, Argentina / Universidad de Buenos Aires, Argentina javier_carol200o@yahoo.com.ar

\author{
Andrés Salanova \\ Universidad de Ottawa, Canadá \\ kaitire@gmail.com
}

\section{Resumen}

El frustrativo, una categoría gramatical presente en varias lenguas sudamericanas y de otras partes del mundo, indica que algún resultado o consecuencia de la eventualidad expresada en la proposición no ha tenido efecto. En un sentido estricto (Overall 2017), señala que la eventualidad se completó, pero sin los efectos esperados; sin embargo, también puede indicar que aquella no llegó a completarse o iniciarse. El examen de los datos de varias lenguas muestra que la alternancia entre estos dos (grupos de) significados es sistemática en la interacción del frustrativo con el aspecto. Así, por ejemplo, en algunas lenguas el frustrativo tiene por defecto el valor de eventualidad completada sin los efectos esperados, y adquiere el valor de eventualidad no completada o iniciada cuando coocurre con morfología imperfectiva, mientras que en otras lenguas ocurre lo inverso: el valor por defecto es el segundo mencionado, y el primero de ellos se obtiene cuando el frustrativo coocurre con morfología perfectiva. En el presente artículo proponemos un análisis formal del frustrativo como aspecto utilizando la noción de inercia (Dowty 1979) a partir, principalmente, de datos de dos lenguas sudamericanas: el chorote (mataguayo, Argentina y Paraguay) y el mẽbengokre (jê, Brasil).

\section{Frustratives as aspect: A view from Chorote (Mataguayan) and Mẽbengokre (Jê)}

\begin{abstract}
The grammatical category of frustrative, present in various languages from South America and other parts of the world, indicates that some result or consequence of the event expressed in a proposition hasn't obtained. In
\end{abstract}

Palabras clave

frustrativo aspecto mataguayo jê

\section{Keywords}

frustrative aspect Mataguayan Jê 
a strict definition (Overall 2017), it shows that the event culminated but without the expected effect; however, it can also indicate that the event was not completed or initiated. The analysis of data from various languages shows that there is a systematic alternation between these two (groups of) meanings when frustrative interacts with aspect. Thus, for instance, in some languages the frustrative morpheme carries by default the meaning of completion without the expected effects, and acquires the value of not culminated or not initiated eventuality when it co-occurs with imperfective morphology. In other languages, the inverse occurs: the default value of frustratives is the latter, while the former is obtained when frustrative co-occurs with perfective morphology. In the present article we propose a formal analysis of frustrative as aspect using the notion of inertia (Dowty 1979), examining primarily data from two South American languages: Chorote (Mataguayan, Argentina and Paraguay) and Mẽbengokre (Jê, Brazil).

\section{O frustrativo como aspecto: Uma análise a partir do Chorote (Mataguaio) e do Mẽbengokre (Jê)}

\section{Resumo}

O frustrativo, uma categoria gramatical presente em várias línguas da América do sul e de outras partes do mundo, indica que algum resultado ou consequência da eventualidade expressada na proposição não ocorreu. Num sentido estrito (Overall 2017), o frustrativo indica que a eventualidade foi completada sem o efeito esperado; no entanto, também pode indicar que ela não foi completada ou mesmo iniciada. A análise de dados de várias línguas mostra que a alternância entre esses dois (conjuntos de) sentidos é sistemática na interação do frustrativo com o aspecto. Desse modo, por exemplo, em algumas línguas o frustrativo tem por defeito o sentido de eventualidade completada sem as consequências esperadas, e adquire o sentido de eventualidade não completada ou iniciada quando ocorre com o aspecto imperfectivo, enquanto que em outras línguas encontra-se o inverso: o sentido por defeito é o segundo, e o primero sentido ocorre quando o frustrativo ocorre com aspecto perfectivo. Neste artigo, propomos uma análise formal do frustrativo como aspecto, empregando a noção de inércia (Dowty 1979), baseando-nos principalmente em dados de duas línguas sulamericanas: o Chorote (Mataguaio, Argentina e Paraguai) e o Mẽbengokre (Jê, Brasil).

\section{Introducción}

Muchas lenguas del mundo, y en particular muchas de América, presentan una categoría que denominaremos aquí frustrativo y que indica que algún resultado o consecuencia de la acción (o estado) no se alcanza (Copley y Harley 2014, Kroeger 2017, Overall 2017). Otras etiquetas que expresan una noción similar son conativo y antirresultativo (cf. Plungian 2001, apud Plungian y van der Auwera 2006). Algunos ejemplos ${ }^{1}$ :

(1) Ruso

$\begin{array}{llll}\text { zrja } & \text { ja } & \text { otkryval } & \text { okno } \\ \text { FRUSTR } & 1 & \text { abrir.IPFV.PAS } & \text { ventana }\end{array}$

'He abierto la ventana (en vano).'

\section{Palavras-chave}

frustrativo aspecto Línguas Mataguaias Línguas Jê

1. Abreviaturas en las glosas: 1,2 , 3: primera, segunda y tercera persona; ac: acusativo; act: activo; al: posesión alienable; antipas: antipasivo; atemp: atemporal; aux: auxiliar; cl: clase; comp: complementante; compl: aspecto completivo; dat: dativo; det: determinante; dup: reduplicación; erg: ergativo; f: femenino; frustr: frustrativo; gen: genitivo; iter: iterativo; h: humano; ipfv: imperfectivo; inm: inmediato; inst: instrumental; intenc: intencional; intns: intensificador; irr: irrealis; mom: momentáneo; $\mathrm{n}$ : nombre; neg: negación; nfut: no futuro; nom: nominativo; nsg: no singular; nvol: modalidad no volitiva; p: adposición; pas: pasado; pas_rec: pasado reciente; pas_rem: pasado remoto; pfv: perfectivo; pl, pl: plural; pos: posesivo; pos_ind: poseedor indefinido; prosp: prospectivo; sg: singular; v: verbo; vact: voz activa; vdat: voz dativa; vi: voz instrumental; vo: voz objetiva; vinst: voz instrumental. 
(2) Tupinambá (Jensen 1998, 539, apud Overall 2017, 480)

a-só-biã

1Sg-ir-FRUSTR

'Fui (pero no conseguí nada).'

(3) Mapudungun (Salas 1992, 131apud Soto y Hasler 2015, 96)

$\begin{array}{llll}\text { Katru"-fu-n } & \text { ñi } & \text { wili } & {[. . .]} \\ \text { cortar-FRUSTR-1Sg } & \text { POS1 } & \text { uña } & \end{array}$

'Me corté las uñas (pero han vuelto a crecer).'

(4) Chorote

\begin{tabular}{|c|c|c|c|}
\hline A-lej-a-ta & ki & i-'yu', & ¡t'ọli’! \\
\hline 1sg.ACT-lavar-MOM-FRUSTR & DET & POS.1sg-ropa & 3.estar_sucio \\
\hline
\end{tabular}

(5) Mẽbengokre

$\begin{array}{llll}\text { Ba } & \text { te } & \text { awỳr } & \text { tẽ } \\ \text { 1NOM } & \text { FRUSTR } & \text { 2.hacia_arriba } & \text { ir }\end{array}$

'Fui a verte [pero no estabas].'

Adoptaremos en este artículo una definición estrecha tomada de un estudio tipológico reciente, según la cual el frustrativo expresa que algún resultado o consecuencia implicado en la proposición no se obtiene (Overall 2017) ${ }^{2}$. Lo que distingue al frustrativo de expresiones adverbiales como en vano es su sistemática variación de significado cuando interactúa con el aspecto. En efecto, los frustrativos de muchas lenguas adoptan otros valores asociados, especialmente los que, siguiendo a Overall (2017), llamaremos incompletivos ${ }^{3}$, que indican acción no completada o interrumpida, y los que indican acción evitada por poco (AEPP en adelante; action narrowly averted en el original inglés). Algunos ejemplos:

(6) Kimaragang (Kroeger 2017, ej. (1b))

$\begin{array}{lllll}\text { Tila'ay } & \text { di=tusing } & \mathrm{i}=\text { pinggan } & \text { pinangakan } & \mathrm{ku} \\ \text { lamer.VDAT.ATEMP } & \text { GEN=gato } & \text { NOM=plato } & \text { VINST.PAS.Comer } & \text { 1sg.GEN } \\ \text { dot } & \text { mangakan } & \text { oku } & \text { po } & \text { dara. } \\ \text { COMP } & \text { VACT.comer } & \text { 1sg.NOM } & \text { aun } & \text { frustr }\end{array}$

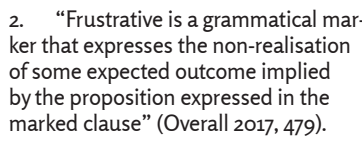

2. "Frustrative is a grammatical marker that expresses the non-realisation of some expected outcome implied by the proposition expressed in the marked clause" (Overall 2017, 479).

3. Lo que aquí llamamos incompletivo puede encontrarse también en la bibliografía bajo el rótulo de conativo. Sin embargo, preferimos evitar este último ya que, al menos en su etimología, remite a la voluntad de un agente (cf. el latín conari 'intentar') y lo que queremos mostrar aquí no involucra necesariamente un agente, véase, por ejemplo, (22).

(7) Tohono O'odham (Copley y Harley 2014, ej (5))

$\begin{array}{lllll}\text { Huan 'o } & \text { cem } & \text { kukpi'ok } & \text { g } & \text { pualt. } \\ \text { Juan AUX.IMPF } & \text { FRUSTR } & \text { open } & \text { DET } & \text { door }\end{array}$

'Juan tiró de la puerta pero no pudo abrirla.'

(8) Kimaragang(Kroeger 2017, ej. (1c))

$\begin{array}{lllllll}\text { Bu-buyuk-an } & \text { no } & \text { dara } & \text { i=iyay } & \text { di=kikina, } & & \\ \text { DUP-engañar-VDAT } & \text { COMPL } & \text { FRUSTR } & \text { NOM=madre } & \text { GEN=chino } & & \\ \text { i } & \text { pinadagang } & \text { do=paray, } & \text { nga } & \text { siri } & \text { oku } & \text { diiri. } \\ \text { NOM } & \text { VACT.PAS.vender } & \text { AC=arroz } & \text { pero } & \text { allí } & \text { 1sg.NOM } & \text { eso }\end{array}$

'Mi mamá \{hubiera sido/estaba por ser\} engañada por el (comerciante) chino cuando vendía el arroz, pero por suerte yo estaba ahí.' 
(9) Chorote

$\begin{array}{lll}\text { A-lej-ta } & \mathrm{ki} & \text { i-'yu'... } \\ \text { 1sg.ACT-lavar-FRUSTR } & \text { DET } & \text { 1sg.POS-ropa }\end{array}$

'Estuve lavando mi camisa...' [No terminé]

'Iba a lavar mi camisa... [No la lavé].

También 'Hubiera lavado mi camisa...'

(10) Mẽbengokre

$\begin{array}{llll}\text { Ba } & \text { bit } & \text { awỳr } & \text { tẽ } \\ \text { 1NOM } & \text { FRUSTR } & \text { hasta.2 } & \text { ir }\end{array}$

'Estaba yendo hasta donde vos estabas' (pero no llegué, porque te encontré en el camino o me volví antes)

(11) Mẽbengokre

$\begin{array}{llllll}\text { Ne } & \text { kam } & \text { bit } & \text { kute } & \text { kanga } & \text { mã. } \\ \text { y } & \text { entonces } & \text { FRUSTR } & \text { 3ERG } & \text { abandonar } & \text { PROSP } \\ \text { 'Y entonces él estaba } & \text { por abandonarla.' }\end{array}$

En este artículo nos concentraremos en las alternancias entre el frustrativo en sentido estricto, ejemplificado en (1)-(5), y el que expresa valores de incompletivo y AEPP, ejemplificado en (6)-(11).Estas alternancias ya han sido identificadas en la bibliografía, y han recibido un análisis en Copley y Harley (2014).

Existen aun otros significados del frustrativo descriptos en la bibliografía pero que no discutiremos aquí:

(12) Otros valores de la morfología frustrativa:

a. Consecuente de un condicional contrafáctico: un evento no ocurrió o no ocurriría porque una condición necesaria no se cumple.

b. "Evaluativo negativo": el hablante está decepcionado por la interrupción de una eventualidad.

c. "Pasado discontinuo": un estado pasado se ha discontinuado.

d. "Optativo": una eventualidad es deseable.

e. "Pasado distante”, usado en narrativas para describir eventos en un tiempo histórico o mitológico.

Algunos de estos valores se documentan también en chorote, una de las lenguas que abordaremos: para (12a) véase la última traducción de (9) arriba y (17) abajo, para(12c) véase (13) y para (12d) véanse (14) -los dos últimos ejemplos con predicados no verbales-y también (24) y (25) abajo. La investigación de estos valores será prioritaria en el futuro.

(13) Chorote

\begin{tabular}{|c|c|c|c|c|c|}
\hline $\begin{array}{l}\text {... Sek } \\
\text { eso }\end{array}$ & $\begin{array}{l}\text { tajl-a-'aja } \\
\text { [3]salir-p-otra_vez }\end{array}$ & $\begin{array}{l}\mathrm{ti} \\
\text { comp }\end{array}$ & $\begin{array}{l}\text { i-li-yi } \\
\text { 3-dejar-P }\end{array}$ & $\begin{array}{l}\text { pa } \\
\text { det }\end{array}$ & $\begin{array}{l}\text { ji-k'yemjla-ta... } \\
\text { 3POS-cónyuge-FRUSTR }\end{array}$ \\
\hline
\end{tabular}

(14) Chorote (var. manjúi, Paraguay)

$\begin{array}{lll}\text { 'Em-ta } & \text { jaja'a } & \text { ka-n-wojme'ne' } \\ \text { por_fin?-FRUSTR } & \text { mucho } & \text { comp-3.IRR-Ilover } \\ \text { ‘Ojalá que llueva!' } & & \end{array}$

El propósito descriptivo del presente artículo es mostrar que existe una relación sincrónica sistemática entre, por un lado, los incompletivos y la 
AEPP y, por otro, los frustrativos en sentido estricto. Esto se lleva a cabo mediante el análisis de datos de dos lenguas sudamericanas, el chorote (mataguayo, Argentina y Paraguay) y el mẽbengokre (jê, Brasil), las cuales ejemplifican dos maneras en las que tal relación puede tener lugar. El propósito teórico es proponer un análisis unificado de los frustrativos que capture su interacción con el aspecto.

\section{Los datos}

\subsection{Chorote}

El chorote es una lengua mataguaya hablada en la provincia argentina de Salta y en el departamento paraguayo de Boquerón. Los datos utilizados en el presente artículo, si no se indica otra cosa, corresponden a la variedad iyojwa'aja', hablada en Argentina, y fueron recogidos por el primer autor en sucesivos viajes al campo entre 2005 y 2015; la traducción de la Biblia citada corresponde a AA. VV. (1997). En esta lengua, la mayoría de las marcas de TAM son sufijos o enclíticos al núcleo predicacional, que no necesariamente es un verbo.

Una de estas marcas, ta(j), indica interrupción de la eventualidad en alguna etapa. Con las atélicas, la interrupción ocurre antes de algún punto contextualmente saliente, típicamente en la etapa de planeamiento y, menos frecuentemente, de desarrollo - véase (15)—, mientras que con las télicas la interrupción significa ausencia de culminación —véase (16)—. En esto, $t a(j)$ contrasta con los progresivos, que focalizan etapas intermedias de la eventualidad, pero no entrañan interrupción o no culminación, sino que esto es solo una inferencia cancelable (cf. con Iba a venir \{pero no vino/y finalmente vino\}).

$(15)$ $\begin{array}{lllll}\text { K'yejli } & \text { a-laki-tye } & \text { ja-kya } & \text { pelóta, } & \text { ton-a-mẹt. } \\ \text { recién } & \text { 1sg.ACT-jugar-FRUSTR } & \text { f-DET } & \text { pelota } & \text { [3]gotear-moM-hacia_acá }\end{array}$ 'Recién estaba por jugar/empezando a jugar a la pelota, y se largó a llover.'

(16)

$\begin{array}{llll}\text { Juan } & \text { nam-ta } & \text { (\#nam } & \text { jiwék). } \\ \text { Juan } & \text { [3]venir-FRUSTR } & \text { [3]venir } & \text { finalmente }\end{array}$

'Juan iba a venir/estaba viniendo (\# y finalmente vino).'

Del párrafo anterior se desprende que, con eventualidades atélicas, la lectura preferida de $t a(j)$ es de AEPP (y, en menor medida, incompletiva) y, con télicas, incompletiva. Sin embargo, es posible que un análisis más profundo muestre que las atélicas siempre dan lugar a una lectura de AEPP, y que los casos de lecturas incompletivas correspondan en realidad a predicados télicos. En efecto, respecto de (15), jugar a la pelota puede conceptualizarse no solo como una actividad sino también como una realización: jugar un partido, i.e. un evento intrínsecamente delimitado, y de la misma manera muchas otras "actividades" pueden volverse predicados télicos. Esto explicaría, entonces, las lecturas incompletivas como la que traducimos por 'recién estaba empezando a jugar'.

Existe una diferencia mínima entre el incompletivo y el condicional contrafactual, ambos expresados mediante $t a(j)$ : 
(17)

$\begin{array}{llcll}\text { 'Nes-ta } & \text { ka } & \text { 'ẹsy-e } & \text { ji-ka } & \text { siwálak. } \\ \text { [3]Ilegar-FRUSTR } & \text { comp } & \text { [3]estar_bien-IRR } & \text { 3pos-al } & \text { bicicleta } \\ \text { 'Habría llegado si su bicicleta hubiera funcionado.' } & & \end{array}$

(18)

'Nes-ta, ji-ka siwálak i-syaj-a.

[3] llegar-FRUSTR 3pos-al bicicleta 3-romperse-p

'Habría llegado/iba a llegar, pero se le rompió la bicicleta.'

Lo interesante en cuanto a la variación sistemática de significado en la interacción con el aspecto se observa cuando ta(j) coocurre con el morfema $a$ ( $e$ tras palatal, ye tras vocal), que llamaremos 'мом(entáneo)' -ver Carol (2011) - y del que consideramos tentativamente que convierte a la eventualidad en delimitada. En tales casos, la eventualidad siempre ocurre efectivamente y, si es télica, culmina, pero sin las consecuencias esperadas. Un par mínimo se observa en (19):

(19)

a.

'Nes-ta.

[3S]llegar-FRUSTR

'Iba a llegar (y no llegó)’.

'Hubiera llegado.'

b.

'Nes-a-ta.

[3S]llegar-MOM-FRUSTR

'Llegó (pero sin los resultados esperados, p.ej. no consiguió lo que vino a buscar).'

Es esencial comprender que las consecuencias esperadas que no se cumplen cuando interactúan $a$ y $t a(j)$ son determinadas contextualmente. No pueden predecirse por el significado del predicado ya que no están codificadas léxicamente. Así, no necesariamente indican interrupción de una actividad o estado, ni la discontinuidad de un estado resultante en las eventualidades télicas: en (19), por ejemplo, el participante continúa en el lugar adonde ha llegado. Algunos ejemplos más:

(20)

\begin{tabular}{llllll} 
A-jlo-ye-ta & wata'a & ka & ijno-'wa-s-e & kyu & \\
1ACT-pedir-MOM-FRUSTR con_esfuerzo & comp & 3.IRR.ir-2-PL-P & un_poco & \\
wat & \multicolumn{1}{c}{ pet } & ka & jl-a'yi-s-a & na-po & si-'lijwa-s, \\
tanto & por_favor? & comp & 3POS-compañero- & DET-PL.h & 1pl.POS- \\
& & & PL-IRR & & paisano-PL \\
'yina & je & ya-kajli. & & \\
pero & neg & 3-poder. &
\end{tabular}

'Le pedí insistentemente que fuera a [verlos a] ustedes junto con los (demás) hermanos, pero no pudo.' (1 Cor 16: 12)

(21)

$\begin{array}{llllll}\text { A-'wen-a-ta } & \text { ta'a } & \mathrm{ki} & \text { in-amtik } & \text { ti } & \text { paj'yi, } \\ \text { 1ACT-ver-MOM-FRUSTR } & \text { ya } & \text { det } & \text { POS.IND-palabra } & \text { comp } & \text { antes } \\ \text { 'nakayi } & \text { pe } & \text { jla'am } & \text { ti } & \text { je } & \text { si-jwey-e. } \\ \text { entonces } & \text { pas_rem } & 3 & \text { comp } & \text { neg } & \text { 1INCT-tener_ánimo-P }\end{array}$


'Ya había conocido la palabra (i.e. el Evangelio) antes, pero en aquel entonces le tenía miedo.'

(22)

$\begin{array}{lllll}\text { Po-ye-ta, } & \text { yina } & \text { ti } & \text { je } & \text { isaj-k'i. } \\ \text { [3] existir-MOM-FRUSTR } & \text { pero } & \text { comp } & \text { neg } & \text { [3]ser_ancho-P }\end{array}$

'[Antes] había [un camino hacia el río], pero no era lo suficientemente ancho.' (Drayson et al. 2000, 96-97)

Para completar la descripción, diremos que $t a(j)$ se liga también a la partícula preverbal de prospectivo ja, la cual a su vez determina la presencia de morfología irrealis en el predicado; la forma resultante es jo-ta(j). Esto da lugar a significados con diversos matices que no podemos detallar aquí, pero todos ellos tienen en común que la eventualidad no llega a comenzar, ni siquiera si es télica, como se ejemplifica en (23), (24) y (25); el primero de ellos da una lectura que puede considerarse de AEPP; el segundo da una apódosis de condicional orientada al futuro o incluso una lectura optativa, cf. (12)d, y el tercero una claramente optativa. (Si bien ta(j) no supone necesariamente una intención - cf. (22), con predicado inagentivotampoco es incompatible con ella, lo que explica una de las traducciones propuestas en (23): 'lo que planeaban hacerle'; es posible que esta lectura se vea favorecida en la combinación con el prospectivo.)

(23)

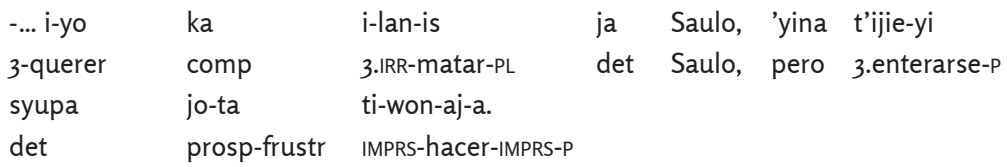

'... decidieron matar a Saulo, pero él se enteró de lo que iban a/planeaban hacerle.' (Hch 9: 23-24)

(24)

$\begin{array}{llllll}\text { Jo-ta } & y \text {-ik } & \text { ka } & \text { tim-e } & \text { ni } & \text { i-sijmaye. } \\ \text { prosp-frustr } & \text { 1ACT.IRR-irse } & \text { comp } & \text { [1ACT.IRR]terminar-P } & \text { det } & \text { 1sg.Pos-trabajo }\end{array}$

'Me iría si terminara mi trabajo.'

(25)

$\begin{array}{lllll}\text { Kilié' } & \text { jo-ta } & \text { tojw-a-'ni } & \text { wat } & \text { pijiá’m } \\ \text { sol } & \text { prosp-frustr } & \text { [3] estar_lejos-IRR-ITER } & \text { muy? } & \text { arriba } \\ \text { ti } & \text { ja-n-laki'n. } & & & \\ \text { comp } & \text { PROSP-3IRR-bailar } & & & \end{array}$

'(Conviene) que el sol siga alto cuando vayan a bailar.' (Drayson et al. 2000, 118-119)

Lo que hace el prospectivo es situar la eventualidad en un tiempo posterior al tiempo tópico o de evaluación; en (23), por ejemplo, si ta(j) se ligara directamente al predicado y no hubiera prospectivo, el significado sería algo como 'se enteró de lo que le estuvieron/estaban haciendo (sin concluirlo)'. En (23) el contexto establece un tiempo de evaluación en el pasado, por lo que el evento "frustrado" - el asesinato de Saulo-puede ser anterior o posterior al tiempo de habla. En (24) y (25), en cambio, al no establecerse contextualmente el tiempo de evaluación, este se sitúa en el presente, lo que parece la opción por defecto para el prospectivo, por lo que el evento "frustrado" o deseable (en las lecturas optativas) se sitúa necesariamente en el futuro deíctico. 


\subsection{Kimaragang}

El kimaragang es una lengua malayopolinesia hablada en el NE de Borneo (Malasia). En ella, el frustrativo exhibe un patrón muy similar al del chorote (Kroeger 2017). La partícula dara indica normalmente que la situación no se ha realizado o no ha culminado, igual que taj según se ve en (6), repetido como (26):

(26)

$\begin{array}{lllll}\text { Tila'ay } & \text { di=tusing } & \mathrm{i}=\text { pinggan } & \text { pinangakan } & \mathrm{ku} \\ \text { lamer.VDAT.ATEMP } & \text { GEN=gato } & \text { NOM=plato } & \text { VINST.PAS.comer } & \text { 1sg.GEN } \\ \text { dot } & \text { mangakan } & \text { oku } & \text { po } & \text { dara. } \\ \text { comp } & \text { VACT.comer } & \text { 1sg.NOM } & \text { aun } & \text { frustr }\end{array}$

'El gato lamió el plato en el que yo comía cuando yo todavía pretendía seguir comiendo.' (Kroeger 2017, ej. (1b))

Sin embargo, cuando el verbo toma una marca explícita de pasado, o bien cuando denota un estado interpretado en presente o pasado ${ }^{4}$, el significado es que la eventualidad ha ocurrido, pero sin los efectos esperados, igual que ataj en chorote:

(27)

Waro dara siin ku nga' n-i-baray ku dot=tutang.

existir frustr dinero 1sg.GEN pero PAS-VINST-pagar 1sg.GEN AC=deuda

'Tenía dinero, pero lo usé para pagar mi deuda.' (Kroeger 2017, ej. (1d))

(28)

$\begin{array}{lcccc}\text { N-o-sii- } \varnothing & \text { ku } & \text { no } & \text { dara } & \text { it=tasu } \\ \text { PAS-NVoL-espantar-vo } & \text { 1sg } & \text { ya } & \text { frustr } & \text { NOM=perro } \\ \text { nga' } & \text { n-iit-an } & \text { oku=i'. } & \\ \text { pero } & \text { PAS-morder-vD } & \text { 1sg=ÉNF } \\ \text { 'Espanté al perro pero me mordió igual.' (Kroeger 2017, ej (1g)) }\end{array}$

Aquí también se observa que la consecuencia esperada (y no actualizada) no necesariamente es la continuidad de la situación o de su estado resultante: esto es así en (27) pero no en (28), donde la expectativa frustrada se refiere a una situación no codificada en el verbo. La explicación que propone Kroeger es similar a la que proponemos para el chorote: el pasado $n$ solo es compatible con una situación efectivizada, de modo que la coocurrencia con el frustrativo dara produce, por coerción, que la situación no actualizada sea otra que se supone derivada de la denotada por el verbo, esto es, de la efectivizada:

since the topic situation cannot [en presencia del morfema de pasado] be interpreted as being unactualized, the "frustrated expectation" interpretation is shifted to an immediate successor situation (Kroeger 2017, 15).

Kroeger conjetura que, en estos casos, el morfema de pasado simplemente indicaría que la situación efectivamente tuvo lugar, y conecta esto con el hecho de que este morfema tiene cognado en otras lenguas de la familia que expresan realis. Nótese que algo muy similar puede decirse del "momentáneo" chorote, cf. (22), donde también parece estar allí simplemente para indicar que el camino efectivamente existía (en ausencia del momentáneo, el significado sería 'hubiera existido' o 'iba a existir').
4. Esta lengua no admite el marcador de pasado cuando el verbo denota un estado, de modo que allí solo el contexto permite la interpretación en pasado (Kroeger 2017, 16).
5. "Dado que la situación tópica no puede [en presencia del morfema de pasado] interpretarse como no actualizada, la interpretación de "expectativa frustrada" se desplaza a una situación inmediatamente sucesora". 
Sugerimos que una interpretación del "pasado" kimaragang como un tipo de perfectivo luce plausible a partir de la comparación con el chorote ${ }^{6}$.

\subsection{El patrón inverso}

Algunas lenguas muestran un patrón que podría considerarse el inverso al que se halla en chorote y kimaragang: en el caso no marcado, el frustrativo indica que la eventualidad se desarrolló o culminó, pero sin las consecuencias esperadas, mientras que la adición de morfología imperfectiva o prospectiva produce lecturas en las que la culminación no se alcanza o la eventualidad no se desarrolla, es decir, lecturas incompletivas o de AEPP. Overall $(2017,489)$ da algunos ejemplos de esto: (29) y (30) muestran eventualidades télicas en las que se excluye la culminación y (31) probablemente una atélica que no llega a iniciarse ${ }^{7}$.

(29) Tariana (Aikhenvald 2003,382)

$\begin{array}{ll}\text { heku-pada-ne } & \text { diwhida } \\ \text { madera-CL:PEDAZO-INST } & \text { 3NSG.f.cabeza }\end{array}$

di-ña-kasu-tha-pidana

3NSG.f-golpear-INTENC-

FRUSTR-PAS_REC.REP

'Estaba a punto de golpearle la cabeza con un pedazo de madera (pero no lo hizo porque el pez se convirtió en una mujer hermosa).'

(30) Urubu-Kaapor (Jensen 1998, 539, apud Overall 2017, 489)

Juka ta tipe
3.matar prosp frustr
'Lo iba a/pensaba matar(pero no lo hizo).'

(31) Sikuani (Queixalós 1991, 21, apud Overall 2017, 489)

pikani ubi-hitsia-nü

frustr plantar-INM-1sg

'Iba a plantar (... y no lo hizo/... pero seguramente no lo hará).'
6. Es cierto que podría considerarse el argumento inverso: que el "momentáneo" chorote a fuera en realidad un realis. En contra de esto puede decirse que $V$ - $a$ suele ser traducido por los hablantes bilingües como 'en ese momentito $V^{\prime}$, generalmente con el verbo en perfecto simple (o bien 'se puso a V'), y que no hay otros contextos, salvo los mencionados, en los que $a$ indique factualidad (es decir, cuya ausencia fuerce o favorezca lecturas contrafácticas). Más aún, es sorprendente que un morfema $a$ ligado a nombres y a cierta clase intermedia entre nombres y verbos expresa, paradójicamente, irrealis. Su posición coincide con la del "momentáneo" y, quizá por haplología, no se documenta coocurriendo con este. 7. Nótese que, si aceptamos los rótulos utilizados en las fuentes de los ejemplos, los morfemas relevantes pertenecen a tres categorías diferentes: modalidad (tariana "intenc(ional)"), tiempo (urubú-kaapor "fut(uro)") y aspecto (sikuani “inm(inencial)"). En base a lo que hallamos en $\mathrm{m}$ bengokre sostendremos que las categorías que interactúan con los frustrativos son primariamente aspectuales.

\subsection{Mẽbengokre}

Una lengua que muestra la interacción descripta en la sección precedente es el mẽbengokre, una lengua jê del Brasil central. El orden de la cláusula es SOV y los modificadores del predicado pueden ser de dos tipos: partículas adverbiales a la izquierda y modificadores léxicos (o cuasiléxicos) a la derecha; estos últimos requieren que el verbo aparezca en su forma nominal.

En muchos dominios, las semánticas de ambos tipos de modificadores se superponen. El frustrativo, por ejemplo, puede expresarse con uno u otro:

(32)

ba te kum arẽ
1nom frustr 3 DAT
'Le conté sin éxito.'


(33)

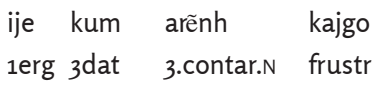

'Le conté en vano (i.e. fue una pérdida de tiempo hacerlo).'

El patrón que nos interesa se ejemplifica en el contraste entre (34), por un lado, y (35) y (36), por el otro. En la cláusula con el frustrativo solo, en (34), la eventualidad se efectiviza, pero sin los resultados esperados, mientras que en (35) y (36), donde el frustrativo coocurre con sendos morfemas aspectuales, la eventualidad no llega a efectivizarse.

(34)

ba te pota 'ãta
1nom frustr puerta abrir.v
'Abrí la puerta...' ('... pero nadie se despertó')

(35)

ba te ije pota ãyr mã

1nom frustr 1erg puerta abrir.n prosp

'Traté en vano de abrir la puerta/Estaba por abrir la puerta [pero me disuadieron].'

(36)

$\begin{array}{lllll}\text { ba te } \quad \text { ije pota } & \text { 'ãyr } & \text { kadjy } \\ \text { 1nom frustr } 1 \text { erg } & \text { puerta } & \text { abrir.N } & \text { intenc } \\ \text { 'Traté de abrir la puerta sin éxito.' } & \end{array}$

Dado que se obtienen lecturas incompletivas y de AEPP cuando el frustrativo se combina con alguno de los enclíticos prospectivos, cabe preguntarse si se podría obtener una lectura estrictamente incompletiva con el progresivo. La construcción progresiva mẽbengokre requiere un auxiliar posicional o de movimiento que subordina una forma nominal del verbo léxico:

(37)

$\begin{array}{llllll}\text { ba ne } & \text { ba } & \text { me } & \text { mã } & \text { i-djujare nh } & \text { o=nhy } \\ \text { 1nom nfut } & \text { 1nom } & \text { pl } & \text { 3.dat } & \text { 1.ANTIPAS.contar.N } & \text { INST=estar_sentado.v } \\ \text { 'Estoy (sentado) contándoles historias.' } & \end{array}$

Sin embargo, la combinación del progresivo con el frustrativo no produce los resultados esperados:

(38)

$$
\begin{aligned}
& \text { ba te pota 'ãyr o=dja } \\
& \text { 1nom frustr puerta abrir.N INST=estar_parado } \\
& \text { 'Estuve abriendo la puerta en vano... (nadie se despertó)' }
\end{aligned}
$$

Estimamos que esto obedece a características de este progresivo en particular, que no excluye significados iterativos con culminación. Aun así, el imperfectivo "gradual" con omõ 'moverse a través de algo' sorprendentemente no puede combinarse con el frustrativo te:

(39)

$$
\begin{array}{lllll}
\text { * ba } & \text { te } & \text { pota } & \text { 'ãyr } & \text { o=mõ } \\
\text { 1nom frustr } & \text { puerta } & \text { abrir.N } & \text { INST=moverse_a_través }
\end{array}
$$


Debemos añadir dos cosas sobre los frustrativos del mẽbengokre. La primera es que existe una partícula adverbial bit -véanse (10) y (11) — que parece estar especializada en el significado de AEPP; al igual que te cuando tiene este valor, requiere el prospectivo. Esta partícula podría ser un desiderativo (la documentación que tenemos de ella es escasa y no disponemos de ejemplos con sujetos no volitivos), pero en cualquier caso muestra un solapamiento significativo con te.

La segunda es que la situación en la cual el objetivo finalmente se logra es admisible con te:

(40)

$\begin{array}{lllllll}\text { ba } & \text { te } & \text { ije } & \text { mar } & \text { mã } & \text { ne arỳm } & \text { kuma } \\ \text { 1nom } & \text { frustr } & \text { 1erg } & \text { saber.N } & \text { prosp y ya } & \text { 3Ac.saber }\end{array}$

'Me esforcé por aprenderlo y [finalmente] lo aprendí.'

Esto choca con la definición propuesta para los incompletivos y con lo que afirmamos abajo sobre el ejemplo (16) del chorote, pero probablemente no sea un contraejemplo serio. La diferencia entre ambos ejemplos podría ser que (40) refiere a dos situaciones distintas, mientras que en (16) las dos eventualidades son parte de una misma situación. En (40), la eventualidad claramente se frustra en la primera situación, más allá de que finalmente el aprendizaje ocurra en la segunda, mientras que el intento de cerrar una puerta ocupa un lapso demasiado breve de tiempo como para que los intentos fallidos y el éxito final se consideren diferentes eventos.

\subsection{Sumario provisional}

Los hechos empíricos del chorote y el mẽbengokre pueden resumirse de la siguiente manera:

a) El chorote ta(j) es incompletivo/AEPP por defecto (con el prospectivo, siempre AEPP) y frustrativo en sentido estricto con мом.

b) El mẽbengokre te es frustrativo en sentido estricto por defecto e incompletivo/AEPP CON PROSP O INTENC.

El tohono o'odham, según la descripción de Copley y Harley (2014), ejemplifica un caso donde el aspecto se marca siempre y donde los imperfectivos, los prospectivos y el perfectivo tienen el mismo efecto sobre la interpretación del frustrativo que en chorote y mẽbengokre (ibíd., ejemplos $(29 a-c))$ :

(41)

Huan 'at o cem kukpi'ok g pualt.

Juan aux.pfv fut frustr abrir det puerta

AEPP: 'Juan intentó/iba a abrir la puerta.' (Tropezó, se arrepintió, etc.)

(42)

Huan 'o cem kukpi'ok g pualt.

Juan aux.ipfv frustr abrir det puerta

Incompletivo: 'Juan intentó abrir la puerta.' (Tiró pero no pudo abrirla) 
(43)

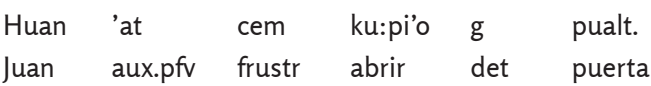

No continuación: 'Juan abrió la puerta pero esta no quedó abierta.'

Objetivo no alcanzado: 'Abrir la puerta no tuvo el efecto esperado.'

Lo que estos datos sugieren es claro: la marcación aspectual en el predicado determina la interpretación del frustrativo. En la próxima sección formalizaremos la interacción entre la interpretación aspectual y el significado del frustrativo. Una hipótesis ulterior sugerida por estos datos es que la diferencia entre el chorote, el mẽbengokre y el tohono o'odham podría atribuirse a propiedades aspectuales inherentes (o por defecto) de los predicados que serían específicas de cada lengua, i.e. algunas lenguas tendrían predicados inherentemente (o por defecto) perfectivos o télicos, mientras que otras los tendrían imperfectivos o atélicos. En su versión más fuerte esta idea no se sostiene, según veremos luego.

\section{Un análisis de los frustrativos como aspecto}

\subsection{Frustrativos e imperfectivo}

Resulta llamativo que por lo menos las lecturas incompletivas y de AEPP puedan ser traducidas por imperfectivos en lenguas como el inglés o el español, que no gramaticalizan el frustrativo. En los ejemplos que siguen, el inglés y el español combinan el imperfecto con la perífrasis prospectiva o la progresiva.

(44)

a. Iba a llegar el viernes pero perdí la conexión.

b. I was getting/going to get here Friday night, but I missed my connection.

(45)

a. Todavía estaba comiendo, pero el gato me lambeteó el plato.

b. I was still eating lunch, but the cat licked the plate.

La principal diferencia entre los frustrativos y los imperfectivos es, según sostenemos aquí, que en un caso se afirma la ausencia de (algún tipo de) culminación y en el otro no. Mientras que los imperfectivos del español o el inglés dejan sin especificar si alguna fase de la eventualidad culmina o no (46), un verdadero frustrativo está fuertemente asociado a la idea de interrupción, como muestra el chorote en (47):

(46) Estaba viniendo para acá cuando me agarró un embotellamiento...

a. ... y por eso no pude llegar.

b. ... pero conseguí desviarme y vine igual.

(47)
Juan nam-ta (\#nam jiwék).
Juan [3]venir-FRUSTR [3]venir finally
'Juan estaba viniendo (\# y finalmente vino).'

Además de los paralelos semánticos entre estos dos tipos de frustrativos y el imperfectivo, hay otros dos hechos que sugieren una asociación del frustrativo con el imperfectivo o, de manera más general, con el aspecto. En 
primer lugar, en mẽbengokre existe un mecanismo de énfasis que consiste en el alargamiento de una sílaba asociada a un tono agudo y que intensifica el valor de un lexema (por ejemplo, adjetivos calificativos). El frustrativo te también puede ser intensificado de esta manera:

(48)

Esta intensificación podría, en principio, afectar el componente frustrativo del significado, i.e. la acción fue decididamente en vano. Sin embargo, lo que sucede en realidad es que la intensificación de te resulta en un significado iterativo o durativo. Otras partículas de alcance por encima de te (contrafáctico, reportativo, futuro) no pueden recibir esta intensificación.

En chorote existe un mecanismo similar que, además del alargamiento y el tono agudo, involucra una articulación "ronca” de la vocal y un sufijo $e$ (o vocal igual a la última de la base si esta termina en $j[\mathrm{~h}]$ ), y tiene un significado similar. La intensificación del frustrativo $t a(j)$ da un resultado algo diferente al documentado en mẽbengokre, ya que indica que la eventualidad que no ocurrió estuvo muy cerca de ocurrir:

(49)

$$
\begin{aligned}
& \text { 'Yal-tá:ja. } \\
& \text { 3.morir-FRUSTR.INTNS } \\
& \text { 'Casi casi se muere.' }
\end{aligned}
$$

Las razones de esta diferencia no nos resultan del todo claras y es posible que estén ligadas a características diferentes de los morfemas frustrativos y/o aspectuales involucrados. De todos modos, el intensificador allí focaliza un intervalo que excluye la culminación, por lo que también allí podría verse una conexión con el aspecto ${ }^{8}$.

En segundo lugar, los predicados marcados con frustrativos tienen características estativas: en narraciones, por lo general, no hacen avanzar el tiempo tópico y se usan frecuentemente para describir eventos que están en segundo plano, o bien para explicar un evento que aparece en primer plano. En mẽbengokre es común encontrar oraciones con frustrativo que repiten otra sin él que sí hace avanzar la narración:

$$
\text { (50) }
$$

$$
\begin{array}{llllll}
\text { Kamy } & \text { jabeje=t } & \text { kum } & \text { akij, } & \text { te } & \text { abeje, } \\
\text { hermano buscar=y } & \text { 3dat } & \text { llamar.v } & \text { frustr } & \text { 3.buscar } \\
\text { te } & \text { kum } & \text { àkjêr } & \text { o=ba. } & & \\
\text { frustr } & \text { 3dat } & \text { 3.llamar.N } & \text { INST=ir } & &
\end{array}
$$

'Buscó a su hermano y lo llamó, lo buscó en vano, anduvo por ahí llamándolo en vano.'

Lo mismo sucede en chorote, donde los frustrativos aparecen a menudo en subordinadas que proporcionan explicaciones, pero no hacen avanzar la narración (véase también el ejemplo (21) arriba):

8. Podría pensarse que la diferencia obedece a distintas características de los predicados: abrir la puerta es agentivo y admite conceptualizaciones como actividad, por lo que en el lapso durante el cual ocurre el intento fallido el agente está haciendo algo y ello habilita la lectura iterativa, mientras que morir es un logro e inagentivo, por lo cual en el lapso análogo el participante no hace nada y por ello la lectura iterativa está vedada. Sin embargo, documentamos esta lectura con actividades como matar (en el sentido de cazar), i.e. 'casi lo maté', 'estuve muy cerca de matarlo (pero no lo logré)'. 
$(51)$

\begin{tabular}{|c|c|c|c|c|}
\hline $\mathrm{Na}$ & i'nyó' & $\mathrm{ti}$ & ji-jwẹlyu’ pọ-ye-ta, & jakjeiyoyiji \\
\hline det & hombre & comp & 3POS-yerno [3S]existir-MOM-FRUSTR & igualmente \\
\hline i-'yen-'ne & ja-pa & jl-ase’. & & \\
\hline
\end{tabular}

\subsection{Inercia, incompletivos y AEPP}

Existe una larga tradición - comenzando con la noción de 'inercia' de Dowty (1979) - de describir a los imperfectivos mediante un componente modal que relaciona una etapa previa a la culminación con la culminación léxicamente codificada en el predicado. De los ejemplos de (44) y (45) se desprende que la inercia que vincula la situación imperfectiva con la culminación del evento debe ser de más de un tipo. Además, las lenguas podrían variar en cuanto al tipo de inercia que favorecen.

En las lenguas eslavas, por ejemplo, una interpretación del imperfectivo que se acerca a (44b) es posible en búlgaro, pero no en polaco (Arregui et.al. 2014, ej. (14)):

(52) Búlgaro

Sledvaštata sedmica pătuvaxme do Pariž, no imaše stački i

próxima.DET semana viajar.IPFV.1pl a París pero haber huelgas y

otkazaxme pătuvaneto.

cancelar.1pl viaje.DET

'íbamos a viajar (lit. 'viajábamos') a París la semana que viene, pero hubo huelgas y suspendimos el viaje.'

(53) Polaco

* Jechaliśmy do Paryża w przyszłym tygodniu,

viajar.IPFV.1pl a Paris en próxima semana

ale teraz są tam strajki więc odwołaliśmy podróż.

pero ahora hay tal huelgas que cancelar.1pl viaje

Significado buscado: 'íbamos a viajar (lit. 'viajábamos') a París la semana que viene, pero hubo tales huelgas que suspendimos el viaje.'

Por otra parte, mientras que en algunas lenguas puede haber un imperfectivo general que sea polisémico, en otras puede haber morfología especializada para imperfectivos con distintas inercias. Este último es el caso del mẽbengokre, donde los imperfectivos con interpretación de futuro relativo se marcan de una manera diferente a la de los progresivos, según se vio en los ejemplos de (35) a (37).

Para dar cuenta de la variación interlingüística, Arregui et al. (2014) proponen que los imperfectivos tienen un "esqueleto" fijo, pero una base modal variable. El esqueleto puede representarse de la siguiente manera:

(54) $[[\mathrm{IPFV}]]=\lambda$ P. $\lambda \mathrm{s} . \forall s^{\prime}: M_{\alpha}(s)\left(s^{\prime}\right)=1 \cdot \exists e \cdot P(e)\left(s^{\prime}\right)=1$ Donde $M_{\alpha}$ es una base modal que toma pares de situaciones que tienen una relación inercial según se define informalmente arriba.

Para las dos lecturas de los imperfectivos de (44) y (45) son necesarios dos tipos de inercia ( $\mathrm{BM}=$ base modal): 
(55) Inercia de Evento [Event Inertia]

$\mathrm{BM}_{\text {inercia-E }}=\lambda s . \lambda s^{\prime}$. $s^{\prime}$ es una situación de Inercia de Evento para $s$, donde, para dos situaciones cualesquiera s y s', s' es una situación de Inercia de Evento para s ssi todos los eventos que efectivamente comenzaron en s continúan en s' como lo harían si no hubiera interrupciones.

(56) Inercia Preparatoria [Preparatory Inertia]

$\mathrm{BM}_{\text {inercia-p }}=\lambda s . \lambda s^{\prime}$. $s^{\prime}$ es una situación de Inercia Preparatoria para $s$, donde, para dos situaciones cualesquiera s y s', s' es una situación de Inercia Preparatoria para s ssi todos los eventos que están en etapas preparatorias en s continúan en s' como lo harían si no hubiera interrupciones.

Para tratar con los frustrativos incompletivos y de AEPP proponemos una leve modificación al esqueleto básico de Arregui et al. (2014) para el imperfectivo, expuesto en (54). En esta nueva versión, diversas inercias pueden alternar para generar las diferentes lecturas del imperfectivo:

(57) $\left[[\right.$ FRUSTR] $]=\lambda P . \lambda s . \forall s^{\prime}: M_{\alpha}(s)\left(s^{\prime}\right)=1 . \exists e: P(e)\left(s^{\prime}\right)=1 \wedge \neg \exists e: P(e)(s)=1$

Donde $M_{\alpha}$ es una base modal que toma pares de situaciones que tienen una relación inercial según se define informalmente arriba.

\subsection{Inercia y frustativo propiamente dicho}

Consideremos ahora lo que sucede con el frustrativo propiamente dicho, es decir, el caso en que la eventualidad efectivamente se completa, pero sin las consecuencias esperadas. La noción de inercia debería ser adecuada para describirlo como lo fue para los incompletivos y de AEPP. En definitiva, la preparación y el desarrollo de P son solamente dos etapas de una secuencia temporal mayor:

(58)

planificación desarrollo culminación consecuencias

Así, puede postularse un tercer tipo de inercia que involucre las consecuencias:

(59) Inercia de Consecuencia [Consequence Inertia]

$\mathrm{BM}_{\text {inercia-c }}=\lambda s . \lambda s^{\prime}$. $s^{\prime}$ es una situación de Inercia de Consecuencia para s, donde, para dos situaciones cualesquiera sys', $s^{\prime}$ es una situación de Inercia de Consecuencia para sssi todos los eventos que se han completado en s son seguidos por las consecuencias esperadas en s' como lo harían si no hubiera interrupciones.

Los imperfectivos con esta base modal no son familiares en las lenguas europeas mejor conocidas. Antes de avanzar en la justificación de por qué sostenemos que esta base modal puede efectivamente hallarse en imperfectivos, realizamos una pequeña digresión para acercar los intentos frustrados a una categoría de significado que sí se halla en los perfectos de algunas lenguas europeas.

Existen casos en los que el estado de cosas que ocurre luego de la culminación o completamiento de un evento parece estar codificado léxicamente. Parsons (1990), por ejemplo, distingue entre estados meta (target states) y estados resultantes (resultant states). En su explicación, algunos predicados tienen especificado en la entrada léxica un estado meta que surge, por 
ejemplo, en las llamadas adjectival passives del inglés (cf. Kratzer 2000), aproximadamente equivalentes a las pasivas con estar en español. Cuando un predicado que tiene especificado un estado meta es seguido por una afirmación de que tal estado ya no se mantiene, la expresión resulta infeliz. Obsérvese el siguiente par del inglés, válido también para algunas variedades de español (nótese que el fenómeno relevante ocurre considerando una lectura de relevancia presente, y no, por ejemplo, de aoristo o experiencial):

(6o)

a. I opened the door, but the wind closed it.

'Abrí la puerta, pero el viento la cerró.'

b. \# I've opened the door, but the wind closed it.

'He abierto la puerta, pero el viento la cerró.'

Cuando no hay un estado meta léxicamente especificado, en cambio, no ocurre tal infelicidad:

(61)

a. I explained this to him, but he forgot.

'Se lo expliqué, pero lo olvidó.'

b. I've explained this to him, but he forgot.

'Se lo he explicado, pero lo olvidó.'

Pese a la ausencia de infelicidad, hay de todos modos un contraste entre (61a) y (61b). La segunda sería indudablemente preferible si uno quisiera expresar frustración ante el hecho de que las consecuencias esperadas de la explicación no ocurrieron. Este es uno de los usos del perfecto de relevancia presente.

Digamos, entonces, que 'relevancia presente' significa que esperamos que algo - la consecuencia relevante en el presente-se siga del hecho de que $P$ ocurrió. En el caso específico de los predicados con estado meta, no hay ambigüedad respecto de lo que esto es (i.e. que el estado meta se mantenga), y de allí la infelicidad de (60b). Pero con los estados resultantes, en cambio, lo esperado está determinado por el contexto conversacional, opción que está disponible incluso para los estados meta: obsérvese este ejemplo también del inglés (con traducción bastante libre al español, donde otra vez el fenómeno se observa en algunas variedades y atendiendo a la lectura de relevancia presente):

(62) A: -Man, it stinks in here.

‘¡Por Dios, qué olor!’

a. B: -l've opened the door already.

'Ya he abierto la puerta.'

b. \# B: -l opened the door already.

'Ya abrí la puerta.'

Nishiyama y Koenig (2010) han reconsiderado recientemente el significado de los perfectos, en particular de los resultativos, y ofrecen para ellos una semántica en la que postulan que existe una situación resultante $X$, inferida pragmáticamente por un principio de informatividad, la cual es posterior a una eventualidad descripta por $\mathrm{P}$, que es la codificada léxicamente:

(63) $\lambda$ s. $\lambda P . \exists e . P(e) \wedge X(s) \wedge \tau(e)<n \wedge \tau(s), n$ 
La diferencia entre los perfectos como el de (61b) y los frustrativos es, naturalmente, que no forma parte del significado de los primeros que las consecuencias esperadas no se sostengan. Es decir, al igual que en el caso del incompletivo y de AEPP, podría postularse que el frustrativo se construye a partir de un significado aspectual. Existen antecedentes de esta idea en la noción de 'antirresultativo' de Plungian (2001) y en la de 'antiperfecto' de Soto y Hasler (2010): los términos sugieren un punto de partida en el resultativo o en el perfecto, a los que se le añadiría la dimensión de interrupción.

\section{4. "Imperfectivos perfectos"}

Podría parecer sorprendente que, por un lado, se describa a los frustrativos con una semántica imperfectiva y, por otro, se postule su proximidad con los perfectos. Sin embargo, no hay contradicción. Pese a que tradicionalmente se ha vinculado a los perfectos con los perfectivos - comenzando por la denominación - no hay razones para suponer una oposición entre perfecto e imperfectivo como la que sí hay entre perfectivo e imperfectivo. De hecho, los perfectos comparten con los imperfectivos -y no con los perfectivos - la característica de tener propiedades estativas (Katz 2003). Otros argumentos sobre la compatibilidad entre perfecto e imperfectivo pueden verse ya en Comrie (1976, 61-64).

Un ejemplo de imperfectivos cuyo significado se superpone ampliamente con el del perfecto puede verse en los llamados imperfectivos factuales del polaco y el ruso, discutidos en detalle en Grønn (2003). La siguiente oración del polaco, de Frąckowiak (2010), se puede interpretar aun sin referente temporal provisto por el contexto y sin avanzar el tiempo narrativo, dos características del perfecto:

(64)

$$
\begin{aligned}
& \text { Jan studiował matematikę c } \\
& \text { Juan estudiar.IPFv matemática.AC } \\
& \text { 'Juan ha estudiado matemática.' }
\end{aligned}
$$

Grønn (2003) discute varios diagnósticos para el perfecto experiencial que se aplican a los imperfectivos factuales, como la coocurrencia, en ruso, con el adverbio kogda-nibud' 'alguna vez', que es incompatible con el pasado perfectivo normal (ibíd., ejemplo (87)):

$(65)$

A ty kogda-nibud’ el $\quad$ odno jabloko na dvoix $\mathrm{s}$ ženščinoj?
y vos alguna.vez comer.IPFV una manzana junto con mujer
‘¿Comiste alguna vez una manzana a medias con una mujer?’

Más importante aún para nuestros propósitos es el hecho de que los imperfectivos factuales tienen implicaciones de relevancia presente, lectura que ha sido llamada "bidireccional" en la bibliografía:

(66)

$$
\begin{aligned}
& \text { a. Ruso } \\
& \text { Vanja priezžal v Moskvu. } \\
& \text { Vanja llegar..PFv in Moscow } \\
& \text { 'Vanja llegó a Moscú' (Grønn’s 2003, ej. (233)) }
\end{aligned}
$$


b. Polaco

Me encuentro con mi amigo Petja y me quejo de que no conozco a nadie que me pueda ayudar a planificar mi viaje a Moscú. La respuesta de Petja es la oración anterior. Entiendo que, debido al hecho de que él llegó previamente a Moscú (aunque no está en Moscú ahora), él puede proveerme la información que necesito. (Adaptado de Frąckowiak 2010).

Lo crucial aquí es que el contexto de (66b) no es uno de estado meta (Vanja no está ahora en Moscú) sino de un estado resultante pragmáticamente inferido (Vanja tiene información útil).

En conclusión, existen todos los elementos para postular que los tipos de frustrativo hallados en chorote y mẽbengokre corresponden a tipos documentados de imperfectivo.

\section{4. ¿Podría ser el frustrativo un modificador de Asp?}

Los hechos observados arriba evidencian interacciones entre la marcación explícita de aspecto y la interpretación del frustrativo. Entonces, cabría preguntarse si no es posible una alternativa a la semántica del frustrativo propuesta en (57): concretamente, una explicación en la cual el frustrativo es un operador que modifica un predicado que ya está aspectualmente modificado. Exponemos la fórmula en (67), donde P es el tipo de las proposiciones y $\mathrm{R}$ es $<\mathrm{P}, \mathrm{P}>$.

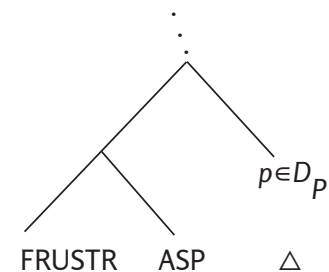

(67) $[[$ FRUSTR] $]=\lambda R \cdot \lambda P . \lambda s \cdot R(P) \wedge \neg \exists e: P(e)(s)=1$

Lo que tal propuesta implicaría es lo siguiente:

(68)

Imperfectivo de eventualidad futura + FRUSTR $\rightarrow$ Acción evitada por poco

Imperfectivo de eventualidad en curso + FRUSTR $\rightarrow$ Incompletivo

Imperfectivo factual + FRUSTR $\rightarrow$ Frustrativo propiamente dicho

Sin embargo, hay algunos problemas empíricos con esto, incluso en nuestros propios datos. El primero de ellos es que el valor del morfema aspectual no siempre se preserva cuando coocurre con el frustrativo: mã es prospectivo por sí mismo en mẽbengokre pero, cuando se combina con el frustrativo, permite lecturas incompletivas y de AEPP, en lugar de solo la primera, según se esperaría si mantuviera su valor.

El segundo es que, si esperamos tal relación directa entre cláusulas marcadas con frustrativo pero sin más marcas aspectuales y las correspondientes sin frustrativo, entonces habría que concluir que las cláusulas sin marca aspectual son siempre perfectivas en mẽbengokre e imperfectivas en chorote. Esto no parece ser el caso, ya que en chorote un predicado télico sin 
marca aspectual (ni modal) se interpreta como concluido en el pasado, mientras que uno de iguales características en mẽbengokre se interpreta como futuro inmediato ${ }^{9}$.

Para dar cuenta de esto último, sería necesario postular ya sea que el frustrativo es imperfectivo por defecto, o bien que solo puede combinarse con un predicado interpretado como imperfectivo (o con cualquier aspecto que tenga la propiedad del subintervalo, a fin de explicar lo que sucede cuando se combina con estativos); en este caso, si el predicado tiene morfología imperfectiva explícita, la función de esta sería especificar si la base modal debe ser factual, en curso, o futura.

\section{Conclusiones y problemas pendientes}

La propuesta elaborada aquí da cuenta de los frustrativo sin completivos y de AEPP con relativamente pocos problemas. El enfoque general puede extenderse para explicar también la no persistencia de los estados meta en los predicados que los codifican, ya que allí el estado meta está especificado léxicamente.

El frustrativo propiamente dicho, en cambio, presenta mayores dificultades, ya que su explicación requiere un tipo de inercia que es problemática. En primer lugar, no se halla en los imperfectivos más comunes. En segundo, no es posible localizar la fase que implica (i.e. la consecuencia de la eventualidad) en los ítems léxicos. Sugerimos que una línea de análisis posible en este sentido es investigar su relación con las llamadas perífrasis escalares o seriales (RAE 2009, 1686-1687 y 2114-2115): en Juan empezó por ponerse/poniéndose de pie se expresa que hay eventualidades que siguen al predicado explicitado pero que no están codificadas léxicamente.

Algunas cuestiones que no han sido profundizadas aquí podrían vincularse con los problemas tratados. Nos referimos concretamente a algunas lecturas del frustrativo mencionadas en la sección introductoria, como las contrafácticas o las de los frustrativos en el futuro, llamadas "optativas" en Kroeger (2017), ya que echarían luz sobre la interacción de los frustrativos con la modalidad y el tiempo. Además, resta explorar la hipótesis de que algunas lenguas podrían tener frustrativos orientados al hablante (lo que explicaría su valor como evaluativo negativo mencionado en la introducción) y otras lenguas los tendrían orientados al sujeto.

Por último,deben mencionarse que la presente propuesta tiene muchos paralelos con la teoría de las "fuerzas" [force-theoretic framework] de Copley y Harley (2014); es posible que sean distintas formalizaciones de un mismo sistema, aunque la inercia y la fuerza parecen ser ontológicamente diferentes. Nos es imposible examinar esto en detalle aquí.
9. En chorote, los predicados sin marca (ni tiempo tópico contextual) se interpretan generalmente en pasado, excepto los estados $y$, a veces, las actividades, que tienden a interpretarse en presente. En m bengokre, los predicados sin marca se interpretan en futuro inminente, excepto los estados, que se interpretan en presente. 


\section{Q Bibliografía}

" Autores Varios. 1997. Sinia' Jlamtis. El Nuevo Testamento en el idioma iyojwa'ja (chorote). Buenos Aires: Sociedad Bíblica Argentina.

"Aikhenvald, Alexandra. 2003. A grammar of Tariana, from Northwest Amazonia. Cambridge: Cambridge University Press.

" Arregui, Ana, María Luisa Rivero y Andrés Pablo Salanova. 2014. "Cross-linguistic variation in imperfectivity". Natural Language and Linguistic Theory 32:307-362.

"Carol, Javier. 2011. "Valores aspectuales y modales de -a y-tajen Chorote (Mataguayo)". Lingüística 26:193-227.

" Comrie, Bernard. 1976. Aspect: An introduction to the study of verbal aspect and related problems. Cambridge: Cambridge University Press.

" Copley, Bridgety; Harley, Heidi. 2014. "Eliminating causative entailments with the forcetheoretic framework: The case of Tohono O'odham cem". En Causation in grammatical structures, editado por Bridget Copley y Fabienne Martin, 120-151. Oxford: Oxford University Press.

" Dowty, David. 1979. Word meaning in Montague grammar. Dordrecht: Reidel.

"Drayson, Nicholas, Sebastian Frías y Julián Gomez. 2000. Sake' iyo ti iyojwa'jats'e’m. Somos chorotes - Nuestras costumbres. Tartagal: ASOCIANA.

" Frąckowiak, Ewelina. 2010. "The semantics of the factual imperfective in Polish and Russian". Manuscrito. Ottawa: University of Ottawa.

" Grønn, Atle. 2003. "The semantics and pragmatics of the Russian factual imperfective". Tesis de doctorado, Universidad de Oslo.

"Jensen, Cheryl. 1998. “Comparative Tupi-Guarani morphosyntax". En Handbook of Amazonian languages, vol. 4, editado por Desmond C. Derbyshire y Geoffrey K. Pullum, 489618. Berlín: Mouton de Gruyter.

" Katz, Graham. 2003. "On the stativity of the English perfect". En Perfect explorations, editado por Artemis Alexiadou, Monika Rathert y Arnim von Stechow, 205-234. Berlín/ Nueva York: Mouton.

" Kratzer, Angelika. 2000. "Building statives". En Proceedings of the Twenty-Sixth Annual Meeting of the Berkeley Linguistics Society: General Session and Parasession on Aspect, 385-399. Berkeley: Berkeley Linguistics Society.

" Kroeger, Paul. 2017. "Frustration, culmination, and inertia in Kimaragang grammar". Glossa: A Journal of General Linguistics 2(1), 56. DOI: http://doi.org/10.5334/gjgl.146.

"Nishiyama, Atsuko, y Jean-Pierre Koenig. 2010. "What is a perfect state?" Language $86: 611-646$.

" Overall, Simon. 2017. "A typology of frustrative marking in Amazonian languages". En The Cambridge handbook of linguistic typology, editado por Alexandra Aikhenvald y R.M.W. Dixon, 477-512. Cambridge: Cambridge University Press.

》Parsons, Terence. 1990. Events in the semantics of English: A study in subatomic semantics. Cambridge: MIT Press.

"Plungian, Vladimir A. 2001. “Antirezul'tativ: do iposlerezul'tata”. En Issledovanija po teorii grammatiki. I: Glagol'nye kategorii, editado por Vladimir A. Plungian, 50-88. Moscú: 
Russkie slovari.

"Plungian, Vladimir A. y Johan van der Auwera. 2006. "Towards a typology of discontinuous past marking”. Sprachtypologie und Universalienforschung 59:317-349.

"Queixalós, Francisco. 1991. Entre cantos y llantos: tradición oral sikuani. Bogotá: Publicaciones de Etnollano.

》Real Academia Española y Asociación de Academias de la Lengua Española. 2009. Nueva gramática de la lengua española. Madrid: Espasa.

》Salas, Adalberto. 1992. El mapuche o araucano: Fonología, gramática y antología de cuentos. Santiago: Centro de Estudios Públicos.

"Soto, Guillermo y Felipe Hasler. 2010. "La no vigencia en el momento de habla: sobre la posibilidad del anti-perfecto como categoría gramatical”. En La renovación de la palabra en el bicentenario de la Argentina, editado por Víctor Castel y Liliana Cubo, 1266-1271. Mendoza: Universidad Nacional de Cuyo.

» Soto, Guillermo y Felipe Hasler. 2015. "El morfema -fu del Mapudungun: la codificación gramatical del antiperfecto". Alpha 40:95-112. 